\title{
Energy Gaps and Interaction Blockade in Confined Quantum Systems
}

\author{
K. Capelle, ${ }^{1,2}$ M. Borgh, ${ }^{2}$ K. Kärkkäinen, ${ }^{2}$ and S. M. Reimann ${ }^{2}$ \\ ${ }^{1}$ Departamento de Física e Informática, Instituto de Física de São Carlos, Universidade de São Paulo, \\ Caixa Postal 369, 13560-970 São Carlos, SP, Brazil \\ ${ }^{2}$ Mathematical Physics, LTH, Lund University, 22100 Lund, Sweden
}

(Received 9 February 2007; published 2 July 2007)

\begin{abstract}
We investigate universal properties of strongly confined particles that turn out to be dramatically different from what is observed for electrons in atoms and molecules. For a large class of harmonically confined systems, such as small quantum dots and optically trapped atoms, many-body particle addition and removal energies, and energy gaps, are accurately obtained from single-particle eigenvalues. Transport blockade phenomena are related to the derivative discontinuity of the exchange-correlation functional. This implies that they occur very generally, with Coulomb blockade being a particular realization of a more general phenomenon. In particular, we predict a van der Waals blockade in cold atom gases in traps.
\end{abstract}

DOI: 10.1103/PhysRevLett.99.010402

Many-body effects in confined systems of interacting quantum particles are a recurring theme, ranging from nuclei over molecules to nanostructured semiconductors [1]. With an initial focus on Bose-Einstein condensation [2], today also the properties of confined fermionic atoms [3] are of concern, and the ability to manipulate trapped atoms recently led to the suggestion of atomtronics [4]. Confinement is often modeled by harmonic-oscillator potentials, but in spite of decades of research the many-body physics in the microscopic as well as the mesoscopic regime is still not fully understood. Even quantities as fundamental as particle-addition and removal energies and energy gaps are hard to calculate if effects of confinement and of particle-particle interactions are of comparable magnitude.

In electronic-structure calculations, addition and removal energies, and gaps are often calculated from density-functional theory (DFT) [5]. A large body of knowledge has been accumulated on how such calculations should be done and when their results are reliable. This knowledge, however, is largely based on the behavior of electrons in atoms, molecules, and solids. Extrapolation to other systems is fraught with dangers and may lead astray in many ways.

In this work, we reassess the calculation of these quantities in confined systems. A key ingredient of our analysis are near-exact ground-state energies, obtained from diagonalizing the many-body Hamiltonian, which allow an unbiased assessment of approximate schemes. As concrete examples, we consider electrons in small quantum dots [1] and fermionic atoms in optical traps [3].

Surprisingly, we find that accurate particle addition and removal energies can be obtained from local-density single-particle potentials, which is not at all what one would expect from experience with atoms, molecules, and bulk semiconductors. From addition and removal energies, we calculate energy gaps and estimate the effect of
PACS numbers: 31.15.Ew, 32.80.Pj, 71.10.- w, 73.21.-b

the derivative discontinuity in confined systems. We relate this discontinuity to Coulomb blockade [6], which allows us to adopt a more general view on blockade phenomena than the usual one, leading, in particular, to the prediction of van der Waals blockade in systems of trapped cold atoms.

To begin with, we define a few basic terms and concepts. The particle-removal energy of an $N$-particle quantum system is defined as

$$
E_{r}(N):=E(N-1)-E(N)=I(N)=-\epsilon_{N}(N),
$$

and the particle-addition energy is given by

$$
E_{a}(N):=E(N)-E(N+1)=A(N)=-\epsilon_{N+1}(N+1) .
$$

Here, $E(N)$ denotes the many-body ground-state energy of the $N$-particle system and $\epsilon_{N}(M)$ denotes the $N$ th eigenvalue of the $M$-particle system. When applied to atoms and molecules, $E_{r}(N)$ and $E_{a}(N)$ become the ionization energy $I$ and electron affinity $A$ respectively. These quantities are defined as differences between ground-state energies pertaining to different systems. In DFT, ground-state energies are readily calculated from the ground-state density, which in turn is obtained from the Kohn-Sham (KS) orbitals. Together with these orbitals, one also obtains KS eigenvalues. Although these are sometimes taken as a zero-order approximation to quasiparticle energies, most of them have no physical significance. The exception to this rule is the energy of the highest occupied state, whose negative is the ionization energy [7-9]. The electron affinity can also be obtained from a highest occupied KS eigenvalue, albeit that of the $N+1$ particle system.

In approximate calculations of ionization energies of atoms and molecules by means of the local-spin-density approximation (LSDA), or by semilocal improvements, it is typically found that values obtained from LSDA total energies agree much better with experiment than do those 
obtained from eigenvalues. The latter can be off by a huge margin [10]. For affinities, the situation is even worse: the LSDA potential often does not even bind one additional electron, predicting instead the anion to be unstable $[12,15,16]$. Commonly, the bad performance of LSDA eigenvalues for predicting ionization energies and electron affinities is attributed to the erroneous asymptotic decay of the LSDA exchange-correlation (XC) potential. For electrons, the exact XC potential (i.e., the one reproducing the exact density) decays as $1 / r$, whereas the potential corresponding to local approximations decays as the density itself, i.e., exponentially. The exponential decay of the LSDA potential means that the outermost electrons are not bound strongly enough, rendering their energies useless for the prediction of electron addition and removal energies. Self-interaction corrections [16] are believed to be required to cure this problem.

The difference between the particle-removal and addition energies is the fundamental energy gap

$$
\begin{gathered}
E_{g}(N):=E_{r}(N)-E_{a}(N) \\
=E(N+1)+E(N-1)-2 E(N)=E_{g}^{\mathrm{KS}}+\Delta_{\mathrm{XC}} \\
=\epsilon_{N+1}(N)-\epsilon_{N}(N)+v_{\mathrm{XC},+}(\mathbf{r})-v_{\mathrm{XC},-}(\mathbf{r}) .
\end{gathered}
$$

Here $v_{\mathrm{XC}, \pm}(\mathbf{r})=\delta E_{\mathrm{XC}}[n] /\left.\delta n(\mathbf{r})\right|_{N \pm \eta}$, with $\eta \rightarrow 0^{+}$is the exchange-correlation (XC) potential calculated at the particle-rich and the particle-poor side of integer particle number $N$. $E_{g}^{\mathrm{KS}}=\epsilon_{N+1}(N)-\epsilon_{N}(N)$ is the $\mathrm{KS}$ gap and $\Delta_{\mathrm{XC}}=E_{g}-E_{g}^{\mathrm{KS}}$ is known as the derivative discontinuity, because in DFT it obeys $\Delta_{\mathrm{XC}}=v_{\mathrm{XC},+}-v_{\mathrm{XC},-}$ [17]. $\Delta_{\mathrm{XC}}$ describes a gap that opens upon addition of a single particle to the system but disappears in the absence of interactions. Because of these characteristics it is, in solids, identified with the Mott gap [17,18].

In quantum dots, the energy gap is traditionally also decomposed into two contributions. The single-particle contribution $\Delta \epsilon$ describes the effects of quantized energy levels due to geometry and confinement. The charging energy $e^{2} / C$ is due to many-body effects which discontinuously raise the energy gap upon addition of one more electron. This effect is hard to describe quantum mechanically, and is therefore typically described phenomenologically, by a classical capacitance $C[1,6,19]$.

Both decompositions of the full gap must add up to the same value, so that $E_{g}^{\mathrm{KS}}+\Delta_{\mathrm{XC}}=\Delta \epsilon+e^{2} / C$. In the phenomenological approach, no general microscopic expressions for $\Delta \epsilon$ and $C$ are given. If $\Delta \epsilon$ is calculated from eigenvalues of noninteracting particles, subject only to the confining potentials, $e^{2} / C$ accounts for all many-body effects. DFT suggests the alternative identification of $\Delta \epsilon$ with the KS gap, and $e^{2} / C$ with $\Delta_{\mathrm{XC}}$. In this case, both $\Delta \epsilon$ and $e^{2} / C$ depend on interaction and particle number, because $E_{g}^{\mathrm{KS}}$ is obtained from the eigenvalues of an effective potential, containing self-consistent Hartree and XC terms.
The alternative identification has the advantage that $E_{g}^{\mathrm{KS}}$ is routinely obtained from DFT codes. The capacitance $C$ then describes the beyond-mean-field contribution to blockade, which need not vanish for $N \gg 1$ [20].

Common local and semilocal-density functionals do not have a discontinuity, and their prediction for the manybody fundamental gap is the KS gap, which can be wrong by a large margin. In principle, however, $\Delta_{\mathrm{XC}}$ can be estimated from eigenvalues obtained by separate calculation of two different systems [21]: combining the righthand sides of Eq. (1) and (2) with Eq. (3), and comparing the result with Eq. (5), one finds

$$
\Delta_{\mathrm{XC}}=\epsilon_{N+1}(N+1)-\epsilon_{N+1}(N),
$$

which allows one to estimate $\Delta_{\mathrm{XC}}$ (defined as a contribution to the many-body gap) even in situations where the functional used to generate the eigenvalues has no discontinuity. Below, we calculate the different contributions to the many-body gap, as well as separate discontinuities and electron addition and removal energies, for electrons in quantum dots as well as for harmonically confined atoms in optical traps.

First, we turn to quantum dots. Here, we consider Coulomb-interacting electrons, which we treat by exact diagonalization and, separately, by the local-spin-density approximation to DFT, in the two-dimensional parametrization of Attaccalite et al. [22]. Representative results for electron removal energies, electron addition energies, and energy gaps are summarized in Tables I and II. Our data for other values of $N$ and $\omega$ (not shown) displayed the same trends.

TABLE I. Negative of the electron-removal energies of a twodimensional $N$-particle dot with confining potential $(1 / 2) m \omega^{2} x^{2}$, obtained exactly $\left(E_{r}^{\mathrm{MB}, \Delta E}\right)$, from LSDA totalenergy differences $\left(E_{r}^{\mathrm{LSDA}, \Delta E}\right)$, and from LSDA eigenvalues $\left(E_{r}^{\mathrm{LSDA}, \mathrm{ev}}\right)$. These are also the negative electron-addition energies of the $(N-1)$-particle systems. (Here and below $\Delta E$ and ev refer to calculations as total-energy differences and from eigenvalues, respectively.) All values are in atomic units. Exact diagonalization data for $\omega=0.01$ are from Ref. [23]; all other data were obtained by us.

\begin{tabular}{ccccc}
\hline \hline$N$ & $\omega$ & $-E_{r}^{\mathrm{MB}, \Delta E}$ & $-E_{r}^{\mathrm{LSDA}, \Delta E}$ & $-E_{r}^{\mathrm{LSDA}, \mathrm{ev}}$ \\
\hline 2 & 0.35 & 0.883 & 0.897 & 1.06 \\
5 & 0.01 & 0.174 & 0.171 & 0.186 \\
& 0.15 & 1.16 & 1.16 & 1.24 \\
& 0.25 & 1.68 & 1.68 & 1.79 \\
& 0.35 & 2.14 & 2.15 & 2.28 \\
6 & 0.15 & 1.35 & 1.33 & 1.41 \\
& 0.25 & 1.92 & 1.91 & 2.02 \\
& 0.35 & 2.44 & 2.43 & 2.56 \\
7 & 0.15 & 1.60 & 1.55 & 1.62 \\
& 0.25 & 2.30 & 2.24 & 2.34 \\
& 0.35 & 2.92 & 2.87 & 2.99 \\
\hline \hline
\end{tabular}


TABLE II. Energy gaps of quantum dots, multiplied by 10 for legibility. See main text for explanation of symbols.

\begin{tabular}{cccclcl}
\hline \hline$N$ & $\omega$ & $E_{g}^{\mathrm{MB}, \Delta E}$ & $E_{g}^{\mathrm{LSDA}, \Delta E}$ & $E_{g}^{\mathrm{LSDA}, \mathrm{KS}}$ & $\Delta_{\mathrm{XC}}$ & $E_{g}^{\mathrm{LSDA}}$ \\
\hline 2 & 0.35 & 5.60 & 5.52 & 2.38 & 2.98 & 5.36 \\
4 & 0.15 & 2.16 & 2.29 & 0.547 & 1.60 & 2.15 \\
& 0.25 & 3.21 & 3.23 & 0.862 & 2.20 & 3.06 \\
& 0.35 & 3.88 & 3.99 & 1.15 & 2.69 & 3.84 \\
5 & 0.01 & 0.349 & 0.297 & 0.0028 & 0.293 & 0.296 \\
& 0.15 & 1.98 & 1.70 & 0.110 & 1.56 & 1.67 \\
& 0.25 & 2.38 & 2.30 & 0.122 & 2.15 & 2.28 \\
& 0.35 & 3.00 & 2.82 & 0.127 & 2.66 & 2.78 \\
6 & 0.15 & 2.46 & 2.16 & 0.654 & 1.46 & 2.12 \\
& 0.25 & 3.76 & 3.30 & 1.22 & 2.00 & 3.22 \\
& 0.35 & 4.80 & 4.39 & 1.84 & 2.45 & 4.29 \\
\hline \hline
\end{tabular}

Several aspects of these results are surprising and unexpected from experience with atoms, molecules, and bulk semiconductors. First, we note in Table I that electron addition and removal energies obtained from eigenvalues are quite close to the exact data. For larger particle numbers, addition and removal energies obtained from LSDA eigenvalues can even be better than those from total-energy differences.

This behavior of addition and removal energies obtained from eigenvalues is dramatically different from a huge body of experience accumulated for atoms, molecules, and solids, where eigenvalue-based ionization energies differ widely from experiment [10], and affinities sometimes cannot be obtained at all [12]. Here, however, the usual explanation of this failure of the LSDA is not applicable: For harmonic confinement of Coulomb-interacting particles, the $1 / r$ behavior of the exact XC potential is still observed, both in three [24] and two [25] dimensions, but the LSDA single-particle orbitals and density now decay as Gaussians. As Fig. 24 of Ref. [1] shows, the self-consistent LSDA density agrees well with the many-body density, even in the asymptotic region. As a consequence, $v_{\mathrm{XC}}^{\mathrm{LSDA}}(\mathbf{r})$ now also decays as a Gaussian, i.e., even faster than exponentially, which could be expected to worsen the performance of the LSDA eigenvalues, instead of improving it.

The effective potential, however, is much stronger than for atoms, because it contains the external confining potential. In a harmonic potential, the system is completely confined; i.e., any number of electrons is bound and there are no continuum states. The absence of continuum states in harmonic confinement, which is a realistic feature of real quantum dots at low energies and atoms in optical traps, is behind the improved binding of the anionlike states, and thus, by means of Eqs. (1)-(6), also behind the other improvements noted. The erroneous asymptotics of the LSDA potential are not sampled by the confined particles.

It is also noteworthy that standard proofs [7-9] of the identification $-\epsilon_{N}(N)=I$ all explicitly or implicitly as- sume that the external potential decays to zero as $|\mathbf{r}| \rightarrow \infty$, or the closely related fact that the single-particle orbitals far away from a finite system decay exponentially with an energy-dependent exponent. Neither is true for harmonic confinement, which grows indefinitely as $|\mathbf{r}| \rightarrow \infty$, and produces single-particle orbitals that decay as Gaussians with a universal (energy independent) exponent. A generalization of the proof to harmonic confinement has been sketched in the appendix of Ref. [26], and is vindicated by our numerical results.

A second aspect of the data that deserves further investigation is the behavior of the gaps in Table II. The LSDA KS gap $E_{g}^{\mathrm{LSDA}, \mathrm{KS}}$, obtained from eigenvalues of an occupied and an unoccupied orbital, greatly underestimates the many-body gap $E_{g}^{\mathrm{MB}, \Delta E}$. This shows that the underestimate arises from the lowest unoccupied orbital, not from any occupied orbital. On the other hand, energy gaps $E_{g}^{\text {LSDA }}$ obtained by adding $\Delta_{\mathrm{XC}}$ to $E_{g}^{\mathrm{LSDA}, \mathrm{KS}}$ (or, equivalently, from differences of eigenvalues pertaining to the $N$-particle and $N+1$-particle KS systems) are only slightly different from LSDA total-energy differences, $E_{g}^{\mathrm{LSDA}, \Delta E}$, being sometimes a bit better and sometimes a bit worse, when compared to $E_{g}^{\mathrm{MB}, \Delta E}$. To within this small fluctuation, manybody gaps of quantum dots can thus be obtained from just two self-consistent calculations of eigenvalues, instead of from three self-consistent calculations of total energies. Note that in these calculations the derivative discontinuity, estimated from Eq. (6), makes a significant contribution to the many-body gap.

Next, we turn to confined fermionic atom gases. The basic formalism of DFT applies both to electrons in quantum dots and cold atoms in traps. The functionals, however, are different because electrons interact via the Coulomb interaction, whereas atoms interact via the van der Waals force. In dilute systems of cold atoms, this is commonly modeled by a suitable contact interaction [27]. Again assuming harmonic external confinement, we perform a Hartree calculation, $v_{\mathrm{XC}} \equiv 0$. The data in Table III show that the atom-removal energies obtained from eigenvalues and from total-energy differences are in similar good agreement for trapped atoms as for electrons in quantum dots, indicating that the key is indeed not the asymptotic behavior of the XC potential, but confinement.

For the trapped atoms, estimates of the derivative discontinuity are also represented in Table III. Not unexpectedly, the discontinuity arising from the contact interaction is smaller than that arising from the much stronger Coulomb interaction, but still makes a significant contribution to the gap, in particular, for larger values of the interaction parameter $g$.

Based on the similarity to the quantum-dot case, we predict that a blockade phenomenon will also occur if repulsively interacting atoms are channeled one by one through an optical trap loaded with a small number of particles and coupled to a reservoir [28]. Interaction-driven 
TABLE III. Negative of the atom-removal energies of $N$ atoms in a harmonic trap with $\omega=1$, interacting with a contact interaction of strength $g$, calculated as total-energy differences and from single-particle eigenvalues, all obtained in the Hartree approximation. These are also the negative atom-addition energies of the $N-1$-particle systems. $E_{g}^{H}$ is the Hartree singleparticle gap, and $\Delta_{\mathrm{XC}}$ is the derivative discontinuity, estimated from single-particle eigenvalues according to Eq. (6). At $N=6$ and $g=1.0$ the exact (CI) gap is 0.9376 , which agrees well with the sum $E_{g}^{H}+\Delta_{\mathrm{XC}}=0.9181$.

\begin{tabular}{cccccc}
\hline \hline$N$ & $g$ & $-E_{r}^{H, \Delta E}$ & $-E_{r}^{H, \mathrm{ev}}$ & $E_{g}^{H}$ & $\Delta_{\mathrm{XC}}$ \\
\hline \multirow{2}{*}{6} & 1.0 & 2.4172 & 2.4328 & 0.8694 & 0.0487 \\
& 2.0 & 2.7748 & 2.8009 & 0.7776 & 0.0902 \\
& 5.0 & 3.6457 & 3.6913 & 0.6113 & 0.1871 \\
10 & 1.0 & 3.4528 & 3.4911 & $4.3 \times 10^{-4}$ & 0.0453 \\
& 2.0 & 3.8537 & 3.9218 & $7.8 \times 10^{-4}$ & 0.0804 \\
& 5.0 & 4.8632 & 4.9963 & $1.6 \times 10^{-4}$ & 0.1569 \\
\hline \hline
\end{tabular}

blockade is not limited to the Coulomb interaction. van der Waals blockade is expected to play a key role in transport experiments on confined cold atoms [28] and in atomtronic devices of the type proposed in Ref. [4].

In conclusion, an unexpected but very favorable scenario emerges from this analysis: many-body particle addition and removal energies of confined systems can be reliably estimated from single-body energies, and the many-body gap and its Coulomb-blockade contribution can be obtained with relative ease and good precision, even from LSDA. These features are expected to apply universally to fully confined systems. Derivative discontinuities give rise to blockade phenomena, which we expect to be ubiquitous, the Mott insulator, Coulomb blockade, and van der Waals blockade being just three particular realizations of a very general phenomenon.

This work was financially supported by the Swedish Research Council, the Swedish Foundation for Strategic Research, the Finnish Academy of Science, and the European Community project ULTRA-1D (No. NMP4CT-2003-505457). K.C. is supported by FAPESP and $\mathrm{CNPq}$, and thanks L. N. Oliveira for useful discussions.

[1] S. M. Reimann and M. Manninen, Rev. Mod. Phys. 74, 1283 (2002).

[2] W. Ketterle, Rev. Mod. Phys. 74, 1131 (2002); E. A. Cornell and C.E. Wieman, Rev. Mod. Phys. 74, 875 (2002).

[3] B. DeMarco and D. S. Jin, Science 285, 1703 (1999).

[4] B. T. Seaman, M. Krämer, D.Z. Anderson, and M.J. Holland, Phys. Rev. A 75, 023615 (2007).

[5] W. Kohn, Rev. Mod. Phys. 71, 1253 (1999).

[6] L. S. Kuzmin and K. K. Likharev, JETP Lett. 45, 495 (1987); T. A. Fulton and G. J. Dolan, Phys. Rev. Lett. 59, 109 (1987); L. S. Kuzmin et al., Phys. Rev. Lett. 62,
2539 (1989); P. Delsing et al., Phys. Rev. Lett. 63, 1180 (1989); 63, 1861 (1989); H. van Houten and C. W. J. Beenakker, Phys. Rev. Lett. 63, 1893 (1989); U. Meirav, M. A. Kastner, and S. J. Wind, Phys. Rev. Lett. 65, 771 (1990); Y. Meir, N. S. Wingreen, and P. A. Lee, Phys. Rev. Lett. 66, 3048 (1991).

[7] C.-O. Almbladh and U. von Barth, Phys. Rev. B 31, 3231 (1985).

[8] J.P. Perdew, R. G. Parr, M. Levy, and J. L. Balduz, Jr., Phys. Rev. Lett. 49, 1691 (1982).

[9] M. Levy, J. P. Perdew, and V. Sahni, Phys. Rev. A 30, 2745 (1984).

[10] As an example, for the $\mathrm{B}$ atom $(Z=5)$ the experimental ionization energy is $0.6099 \mathrm{Ry}$. The LSDA prediction from total energies is $0.6294 \mathrm{Ry}$, whereas the LSDA prediction from the highest occupied eigenvalue is $0.2997 \mathrm{Ry}$, which is off by a factor of 2 (data from Ref. [11]).

[11] M. P. Lima et al., J. Chem. Phys. 126, 144107 (2007).

[12] As an example, for the $\mathrm{F}$ atom $(Z=9)$ the experimental electron affinity is $3.40 \mathrm{eV}$. For common basis sets, the LSDA prediction from total energies is $4.63 \mathrm{eV}$, whereas the LSDA prediction from the highest occupied eigenvalue is $-0.03 \mathrm{eV}$, which wrongly indicates an unbound anion (data from Ref. [13]). In basis sets specially designed to bind the extra electron, the latter value changes to $1.5 \mathrm{eV}$, which is bound, but still a factor of 3 off the experimental value [14].

[13] J. M. Galbraith and H. F. Schaefer, III, J. Chem. Phys. 105, 862 (1996).

[14] A. A. Jarecki and E. R. Davidson, Chem. Phys. Lett. 300, 44 (1999).

[15] N. Rösch and S.B. Trickey, J. Chem. Phys. 106, 8940 (1997).

[16] C.D. Pemmaraju, T. Archer, D. Sánchez-Portal, and S. Sanvito, Phys. Rev. B 75, 045101 (2007); J. P. Perdew and A. Zunger, Phys. Rev. B 23, 5048 (1981).

[17] J.P. Perdew and M. Levy, Phys. Rev. Lett. 51, 1884 (1983); L. J. Sham and M. Schlüter, Phys. Rev. Lett. 51, 1888 (1983).

[18] N. A. Lima, L. N. Oliveira, and K. Capelle, Europhys. Lett. 60, 601 (2002).

[19] M. A. Kastner, Phys. Today 46, No. 1, 24 (1993).

[20] The Mott insulator, from this point of view, is a consequence of blockade on an atomic scale, instead of a nanoscopic one, occurring between neighboring minima of the crystal potential instead of dots.

[21] F.P. Rosselli, A.B.F. da Silva, and K. Capelle, arXiv:physics/0611180.

[22] C. Attaccalite, S. Moroni, P. Gori-Giorgi, and G. B. Bachelet, Phys. Rev. Lett. 88, 256601 (2002); 91, 109902(E) (2003).

[23] M. Rontani et al., J. Chem. Phys. 124, 124102 (2006).

[24] K.-C. Lam, F. G. Cruz, and K. Burke, Int. J. Quantum Chem. 69, 533 (1998).

[25] I. D'Amico and G. Vignale, Phys. Rev. B 59, 7876 (1999).

[26] J. P. Perdew and M. Levy, Phys. Rev. B 56, 16021 (1997).

[27] K. Kärkkäinen, M. Borgh, M. Manninen, and S.M. Reimann, New J. Phys. 9, 33 (2007).

[28] A. Recati, P. O. Fedichev, W. Zwerger, J. von Delft, and P. Zoller, Phys. Rev. Lett. 94, 040404 (2005). 